Review

\title{
Does Religiosity Predict Suicidal Behavior?
}

\author{
David Lester \\ Department of Psychology, Stockton University, Galloway, NJ 08205-9441, USA; david.lester@stockton.edu
}

Received: 22 August 2017; Accepted: 23 October 2017; Published: 31 October 2017

\begin{abstract}
Research was reviewed on whether self-report measures of religiosity were a protective factor against suicidal behaviors. It was found that scores on Francis's measure of religiosity was negatively associated with non-lethal suicidal behavior (ideation and attempts), a protective effect. Similarly, it was found that intrinsic religiosity (but not extrinsic religiosity) was negatively associated with non-lethal suicidal behaviors. However, these associations were weak. Research is needed on the issue whether counselors can use their patients' religiosity to reduce the risk of dying by suicide.
\end{abstract}

Keywords: religiosity; suicidal ideation; attempted suicide

The association of religion and suicide has long been of interest. Durkheim (1897) noted and tried to explain the fact that Protestants have higher suicide rates than Roman Catholics, while it has long been known that Muslims have lower suicide rates than Christians (Lester 2006). There has been a great deal of research and theorizing about the role of religion, religiosity, and spirituality in increasing the risk of suicidal behavior versus acting as a protective factor for suicidal behavior. ${ }^{1}$

In a meta-analysis of nine studies, Wu et al. (2015) found significant protective effects of religion (as measured by church/temple attendance or claiming/practicing a religion) on completed suicide. This effect was found to be stronger in studies conducted in Western cultures, in areas with high religious homogeneity, and among older populations. Sisask et al. (2010) also reported cross-cultural differences in the protective effects of three dimensions of religiosity (religious denomination, subjective religiosity, and organizational religiosity) for attempted suicide. The strongest protective effect across the different study sites, such as Brazil, Estonia, the Islamic Republic of Iran, and Sri Lanka, was found for subjective religiosity, that is, considering oneself to be a religious person, while the results for the more objective indicators of religiosity were mixed. The present chapter focuses on the association between religiosity and suicidal behavior in individuals. Since the research on this association involves self-report questionnaires, ${ }^{2}$ the suicidal behavior is limited to suicidal ideation and, occasionally attempted suicide. Furthermore, we will focus more on our own research, but not to the exclusion of the research of others.

\section{Religiosity, Subjective Well-Being and Mental Health}

There has been a great deal of research on religiosity and its correlates, in recent years. For example, many studies report that religiosity is associated with subjective well-being (e.g., Abdel-Khalek 2012b; Greene and Yoon 2004), although occasional studies report a failure to find an association (e.g., Lewis 2002). Although most of the research has been carried out on European and American samples, research on Arab Muslim samples have also reported positive associations between religiosity and subjective well-being (e.g., Abdel-Khalek 2012a).

1 For recent reviews of some of this scholarly literature, see Colucci and Martin (2008), Krysinska and Lester (2016) and Lawrence et al. (2016).

2 We will not include measures such as church attendance, participation in religious activities, and other behaviors, but only that using formal scales of religiosity, such as that developed by Francis (1992), and single-item self-report measures of religiosity. 
Religiosity is also associated with mental health. A recent meta-analysis of 32 studies on the association between religiosity and anxiety in Arab samples (Abdel-Khalek et al. forthcoming) found that all the studies reported a negative association, and all but one were statistically significant. Biegel and Lester (1990) found that religiosity was negatively associated with neuroticism, as measured by the Eysenck Personality Inventory (Eysenck and Eysenck 1963) but, again, not all studies report this negative association (e.g., Rife and Lester 1997).

In a study of 460 Kuwaiti students and 274 American students, Abdel-Khalek and Lester (2007) found that self-reported religiosity was positively associated with self-reported mental health in both groups. In a second study of 158 American students and 192 Kuwaiti students, Abdel-Khalek and Lester (2010) reported that religiosity was positively associated with happiness, love of life and self-rated mental health and negatively with anxiety ${ }^{3}$ and with depression in both samples of students ${ }^{4}$. In a third study, this time of 154 Kuwaiti students and 154 American students, Abdel-Khalek and Lester (2013) reported that, for the Kuwaiti students, religiosity scores were positively associated with scores on a formal scale of mental health and self-rated mental health, optimism, and self-esteem, and negatively with pessimism and hopelessness. For the American students, only the correlations with scores on the mental health scale, self-rated mental health, and self-esteem were statistically significant.

On the whole, therefore, individuals who score high on measures of religiosity seem to be happier and to have better mental health.

\section{Religiosity and Suicidality}

Religiosity and spirituality are generally recognized to be protective factors for suicidal behavior. Research and clinical evidence have suggested that religiosity can be linked to a lower risk of suicidal ideation, suicide attempts, and completed suicide (Moreira-Almeida et al. 2006). A number of theories and models have been proposed to explain the salutary effect of religious and spiritual beliefs, behaviors, and social networks, including the social networks created by involvement in religious activities (which increase social integration) and the hope created by religious beliefs (such as a belief in an afterlife) (Stack and Kposowa 2011).

Systematic reviews of the literature, and general population studies, find strong evidence regarding the protective role of many aspects of religiosity against suicide. About three-quarters of the studies published to-date, including studies in Western (predominantly Christian) and in Middle Eastern (predominantly Muslim) countries, have reported significant inverse relationships between at least one aspect of religion and at least one dimension of suicidality (Koenig et al. 2012; Stack and Kposowa 2011). A meta-analysis of international studies, including case-control and retrospective cohorts, found an overall protective effect of religiosity against suicide (Wu et al. 2015).

Most studies of "religiosity" use a variety of measures, including religious affiliation and church attendance. The present chapter will review research using three measures: Francis's religiosity scale (Francis 1992), the intrinsic-extrinsic religiosity scale (Gorsuch and McPherson 1989) and a single-item self-report measure (Abdel-Khalek and Lester 2007).

\section{Francis's Religiosity Scale}

Francis (see Lester and Francis 1993) has developed a scale to measure religiosity which is composed of a heterogenous collection of items, assessing religious beliefs and behaviors. Krysinska and Lester (2016) and Krysinska et al. (2015) gave American undergraduate students Francis's religiosity scale, a depression scale, and questions about their history of suicidal ideation threats and attempts, and current suicidal ideation. They also administered a brief version of Linehan's Reasons for Living Scale (Osman et al. 1996) which has subscales that measure survival and coping

3 Not significant for Kuwaiti students.

4 Not significant for American students. 
beliefs, responsibility to family, moral objections, fear of suicide, and fear of social disapproval. Religiosity scores were associated with less lifetime suicidal ideation (but not lifetime suicide attempts or current suicidal ideation). However, in multiple regressions, religiosity scores did contribute to the prediction of lifetime suicide attempts, along with gender and moral objections to suicide and responsibility to family. Thus, the role of religiosity in this study was present but weak and inconsistent.

It is important, in research, to control for possible confounding variables. Lester and Francis (1993) examined the association between religiosity and past and current suicidal ideation in American undergraduate students. The association was negative for both past and current suicidal ideation. These associations remained in multiple regressions with depression alone and with belief in an internal-external locus of control alone, but not when religiosity was combined with extraversion, neuroticism, and psychoticism scores using Eysenck's scale (Eysenck et al. 1985).

A summary of the results of this research is presented in Table 1. Of the five correlation coefficients, all five are negative, and two are statistically significant using two-tailed tests of significance.

Table 1. Research using Francis's Religiosity Scale (Pearson correlations shown).

\begin{tabular}{cccc}
\hline Study & Variable & Pearson $\mathbf{r}$ & $\mathbf{n}$ \\
\hline \multirow{2}{*}{ Lester and Francis (1993) } & $\begin{array}{c}\text { current suicidal ideation } \\
\text { past suicidal ideation }\end{array}$ & -0.18 & $\mathrm{n}=103$ \\
\hline Krysinska et al. (2015) & lifetime suicidal ideation & $-0.21 *$ & $\mathrm{n}=165$ \\
\hline \multirow{3}{*}{ Krysinska and Lester (2016) } & lifetime suicidal threat & -0.03 & \\
& lifetime suicide attempt & -0.01 & \\
& current suicidal ideation & -0.10 & \\
\hline & ${ }^{*}$ Two-tailed $p<0.05$. &
\end{tabular}

\section{Intrinsic-Extrinsic Religiosity}

Whereas Francis's religiosity scale contains a heterogenous selection of items, Gorsuch and McPherson (1989) published a scale that measures two major aspects of religious beliefs and behaviors. Intrinsic religiosity concerns direct engagement with religion, such as belief in the tenets of the Christian religion and praying, whereas extrinsic religiosity concerns the indirect benefits of engagement, such as the social relationships formed as a result of church attendance.

In a study of American undergraduate students, Lester (2012) compared measures of religiosity and spirituality as predictors of past suicidal ideation and attempts (measured using items from Thalbourne's manic-depressive scale (Thalbourne et al. 1994)) ${ }^{5}$. For religiosity, the intrinsic-extrinsic religiosity scale (Gorsuch and McPherson 1989) was used. For spirituality, a scale devised by Hall and Edwards (2002) was used, which measures awareness (e.g., I have a sense of how God is working in my life), instability (e.g., My emotional connection with God is unstable), grandiosity (e.g., God recognizes that I am more spiritual than most people), and impression management (e.g., I am always in a worshipful mood when I go to church). A history of suicidal ideation was positively associated with intrinsic religiosity, but not significantly with extrinsic religiosity, and positively associated with the awareness and instability subscale scores of spirituality, but not significantly with grandiosity and impression management subscale scores. In a multiple regression, a history of suicidal ideation was predicted by depression (positively), extrinsic religiosity (negatively), helplessness (positively), and awareness (positively). The positive association of a history of suicidal ideation and intrinsic religiosity is contrary to the results of other studies, but is consistent, in this study, with the positive association with the awareness component of spirituality. The association of a history of suicidal ideation with the instability component of spirituality is more in line with expectations

5 Past suicide attempts were not common, and not significantly associated with any of the scale scores. 
from previous research. Incidentally, in Lester's study, a measure of depression was also positively associated with intrinsic religiosity and unstable spirituality. The measures of spirituality, but not the measures of religiosity, contributed to the prediction of depression in a multiple regression. ${ }^{6}$

Walker and Bishop (2005) administered measures of suicidal ideation, depression, intrinsicextrinsic religious orientation and social support to American university students. Overall, suicidal ideation scores (measured using the Beck Scale for Suicide Ideation (Beck and Steer 1993)) were positively associated with depression scores, and negatively associated with intrinsic religiosity scores and social support scores, and this was found for both White and for African American students. Lester and Walker (2017) obtained results which conflict with this. Lester and Walker examined the association between religiosity (using the intrinsic/extrinsic religiosity scale) and scores on Reynolds' (1987) scale to measure current suicidal ideation. For the total sample of students, both intrinsic and extrinsic religiosity scores were negatively associated with suicidal ideation scores. Only intrinsic religiosity scores predicted suicidal ideation in a multiple regression (along with depression and hopelessness scores). However, this association was statistically significant for only the European females but, if entered along with depression and hopelessness scores in a multiple regression, the impact of religiosity was no longer statistically significant for European females. ${ }^{7}$

Walker et al. (2014) studied 236 African American men and women. Suicidal ideation, measured using Reynolds' (1987) scale, was negatively associated with intrinsic religiosity but not with extrinsic religiosity. However, for individuals with low scores for extrinsic religiosity, perceived racism played a significant role in predicting suicidal ideation (mediated through depression). Thus, extrinsic religiosity buffered the effects of racism on suicidal ideation.

Hovey et al. (2014) found that only intrinsic religiosity was associated with suicidality (as measured by the Suicide Behaviors Questionnaire: Osman et al. 2001) in a sample of American undergraduates but, in a multiple regression, intrinsic religiosity was no longer statistically significant as a predictor of suicidality, outweighed by a scale to measure perceived emotional support from church members.

Ji et al. (2011) obtained a sample of 11,481 high school students in schools adhering to an evangelic church in Canada and the United States, and measured suicidality by averaging answers to two questions, one about how often they had been depressed in the past month, and how often they had attempted suicide in their lifetime. Intrinsic religiosity was a stronger correlate of depression/suicidality than was extrinsic religiosity $(-0.15$ versus +0.09$)$, and had a protective effect. ${ }^{8}$

Hill and Francis (2005) found that neither intrinsic religiosity, nor extrinsic religiosity were associated with scores on a scale of past and present suicidality in a sample of 501 undergraduates at a religiously-oriented college in Wales (UK). ${ }^{9}$

Liu and Koenig (2013) studied 1039 rural Chinese women, and found that intrinsic religiosity, as measured by Hoge's (1972) scale, was negatively associated with scores on Beck's Suicide Ideation Scale.

A summary of these results is shown in Table 2. For intrinsic religiosity, 6 of 8 correlations were negative, of which 5 were statistically significant. For extrinsic religiosity, 4 of 7 correlations were negative, one of which was statistically significant. However, one positive correlation was also statistically significant. Thus, the association of intrinsic religiosity proved to be a more consistent protective factor for suicidal behavior. It appears, therefore, that commitment to the beliefs and rituals of Christianity is more of a protective factor for suicidal behavior than the indirect benefits, such as the social relationships that might develop from regular church attendance.

6 A study by Wang et al. (2009) found that higher scores on Hall and Edwards (2002) spirituality assessment scale were associated with lower suicidal ideation in black male students, but not in black female students, suggesting an important role of sex, and perhaps ethnicity, in these associations.

7 Of interest is the fact that both depression and hopelessness scores predicted suicidal ideation for both European males and females, but only depression scores predicted suicidal ideation for African American males and females.

8 Ji et al. found that extrinsic religiosity was associated with increased depression/suicidality, substance abuse and delinquency.

9 Hill and Francis report multiple regressions, but did not report the Pearson correlations. 
Table 2. Research using intrinsic-extrinsic religiosity scales (Pearson correlations shown).

\begin{tabular}{|c|c|c|c|}
\hline Study & Intrinsic Religiosity & Extrinsic Religiosity & $\mathbf{n}$ \\
\hline \multicolumn{4}{|l|}{ Hill and Francis (2005) } \\
\hline past and current suicidality & na & na & 501 \\
\hline \multicolumn{4}{|l|}{ Walker and Bishop (2005) } \\
\hline suicide ideation & $-0.20 *$ & -0.06 & 459 \\
\hline \multicolumn{4}{|l|}{ Ji et al. (2011) } \\
\hline depression/prior attempts & $-0.15^{*}$ & $+0.09 *$ & 11,481 \\
\hline \multicolumn{4}{|l|}{ Lester (2012) } \\
\hline past suicidal ideation & +0.16 & +0.05 & 149 \\
\hline past suicide attempts & +0.10 & -0.03 & \\
\hline \multicolumn{4}{|l|}{ Liu and Koenig (2013) } \\
\hline suicide ideation & $-0.22 *$ & & 1039 \\
\hline \multicolumn{4}{|l|}{ Hovey et al. (2014) } \\
\hline suicide behaviors & -0.13 & +0.03 & 200 \\
\hline \multicolumn{4}{|l|}{ Walker et al. (2014) } \\
\hline suicidal ideation & $-0.22 *$ & -0.09 & 236 \\
\hline \multicolumn{4}{|l|}{$\begin{array}{l}\text { Lester and Walker (2017) } \\
\text { suicide ideation }\end{array}$} \\
\hline all Ss & $-0.22 *$ & $-0.14 *$ & 419 \\
\hline women & $-0.21 *$ & $-0.16 *$ & \\
\hline men & -0.19 & -0.07 & \\
\hline African Americans & -0.09 & -0.04 & \\
\hline European Americans & $-0.21 *$ & $-0.16^{*}$ & \\
\hline African American women & -0.09 & -0.08 & \\
\hline African American men & -0.04 & +0.18 & \\
\hline Eur. American women & $-0.20 *$ & $-0.18 *$ & \\
\hline Eur American men & -0.21 & -0.10 & \\
\hline
\end{tabular}

${ }^{*}$ Two-tailed $p<0.05$; na: not available.

\section{Single-Item Measures}

In their study, mentioned above, of Kuwaiti and American college students, Abdel-Khalek and Lester (2007) found that self-reported religiosity (using an item "What is your level of religiosity in general") was negatively associated with suicidal ideation (measured using Reynolds' (Reynolds 1987) Suicidal Ideation Scale) in both groups.

\section{Studies of Clinical Populations}

Caribé et al. (2015) studied 164 psychiatric outpatients in Brazil with bipolar disorder Type 1. Those reporting at least one lifetime suicide attempt scored lower on a measure of intrinsic religiosity (Lucchetti et al. 2012) than those not reporting a suicide attempt, even after controls for gender, employment, presence of a partner, children, psychiatric comorbidity, rapid cycling, family history of attempted suicide and of completed suicide, and impulsivity.

Caribé et al. (2012) compared 110 suicide attempters presenting at a Brazilian hospital with control subjects chosen from family members accompanying the suicide attempter (which controlled for socio-economic variables). The suicide attempters reported significantly lower levels of intrinsic religiosity (and, in addition, organizational and non-organizational religious activities) than the control subjects. This difference remained significant even after controlling for mental illness, impulsivity and sociodemographic variables.

\section{Discussion}

Study of the association between religiosity and suicidal behavior has been confused in the past by the different measures of religiosity employed in the research. Some studies have used religious affiliation, others religious behavior (such as church attendance and praying), while still others have 
used reliable and validated religiosity scales. Some recent research has focused on "spirituality" rather than "religiosity".

The present review focuses on research using validated scales of religiosity. For Francis's scale of religiosity, religiosity proved to be a consistent, but weak, protective factor against suicidal ideation and attempts. For the different measures of intrinsic/extrinsic religiosity, intrinsic religiosity proved to be a consistent, but weak protective factor against suicidal ideation and attempts. Associations between extrinsic religiosity and suicidal behavior were inconsistent.

These results suggest that religious behaviors, which are often used as a measure of "religiosity", are most likely less relevant as protective factors against suicidal behavior because these religious behaviors overlap with the items used to measure extrinsic religiosity. However, it seems necessary for future research to devise more precise measures of "religiosity" beyond that provided by the intrinsic/extrinsic dichotomy. In particular, a distinction might be made between beliefs and behaviors, for example, a belief in God versus praying.

Furthermore, a belief in God may entail a variety of sub-beliefs. For example, some of those who believe in a Christian God may believe that suicide is a sin, and that they will be punished after death. A few believe that, if a person dies by suicide, then he or she will be reincarnated to live out those years that were missed, as a result of the premature death by suicide. Others may believe that God will understand their situation and forgive them. Jacobs (1967) in his analysis of suicide notes, found that a common form for suicide notes specified that the problems faced by the individual are unbearable, asked God for forgiveness, and requested others to pray for his soul. It is therefore critical, for a meaningful study of the association between religiosity and suicidal behavior, to ascertain the individuals' beliefs about suicide in relation to Christianity.

The question arises whether counselors and psychotherapists can use the religiosity of those whom they counsel to decrease the risk of suicidal behavior in their clients. There is no research on this but, since most religions view suicide as an inappropriate behavior (a sin), religiosity ought to be a strong protective factor against dying by suicide, even if it is less effective against suicidal ideation and perhaps suicide attempts.

Conflicts of Interest: The author declares no conflict of interest.

\section{References}

Abdel-Khalek, Ahmed M. 2012a. Associations between religiosity, mental health, and subjective well-being among Arabic samples from Egypt and Kuwait. Mental Health Religion \& Culture 15: 741-58.

Abdel-Khalek, Ahmed M. 2012b. Subjective well-being and religiosity. Mental Health, Religion and Culture 15 : 39-52.

Abdel-Khalek, Ahmed M., and David Lester. 2007. Religiosity, health, and psychopathology in two cultures. Mental Health, Religion \& Culture 10: 537-50.

Abdel-Khalek, Ahmed M., and David Lester. 2010. Constructions of religiosity, subjective well-being, anxiety, and depression in two cultures. International Journal of Social Psychiatry 58: 138-45.

Abdel-Khalek, Ahmed M., and David Lester. 2013. Mental health, subjective well-being, and religiosity. Journal of Muslim Mental Health 7: 63-76.

Abdel-Khalek, Ahmed M., L. Nuño, J. Gómez-Benito, and David Lester. Forthcoming. A meta-analysis of the relationship between religiosity and anxiety based on 32 Arabic studies $(N=15,207)$.

Beck, Aron T., and Robert Steer. 1993. Beck Scale for Suicide Ideation. San Antonio: Psychological Corporation.

Biegel, Kenneth, and David Lester. 1990. Religiosity and psychological disturbance. Psychological Reports 67: 874.

Caribé, André C., R. Nunez, D. Montal, L. Ribeiro, S. Sarmento, L. C. Quarantini, and Â. Miranda-Scippa. 2012. Religiosity as a protective factor in suicidal behavior: A case-control study. Journal of Nervous and Mental Disease 200: 863-67.

Caribé, André C., P. Studart, S. Bezerra-Filho, E. Brietzke, M. N. Noto, M. Vianna-Sulzbach, F. Kapczinski, F. Silva Neves, H. Correa, and Â. Miranda-Scippa. 2015. Is religiosity a protective factor against suicidal behavior in bipolar I outpatients? Journal of Affective Disorders 186: 156-61. 
Colucci, Erminia, and Graham Martin. 2008. Religion and spirituality among the suicidal path. Suicide $\mathcal{E}$ Life Threatening Behavior, 38: 229-44.

Durkheim, Emile. 1897. Le Suicide. Paris: Felix Alcan.

Eysenck, Hans Jurgen, and S. B. G. Eysenck. 1963. The Eysenck Personality Inventory. San Diego: EDITS.

Eysenck, S. B. G., Hans Jurgen Eysenck, and P. Barrett. 1985. A revised version of the psychoticism scale. Personality $\mathcal{E}$ Individual Differences 6: 21-29.

Francis, Leslie J. 1992. Reliability and validity of the Francis Scale of Attitude towards Christianity. Panorama 4 : 17-19.

Gorsuch, Richard L., and Susan E. McPherson. 1989. Intrinsic/extrinsic measurement. Journal for the Scientific Study of Religion 28: 348-54.

Greene, Kenneth V., and Bong Joon Yoon. 2004. Religiosity, economics and life satisfaction. Review of Social Economy 62: 245-61.

Hall, Todd W., and Keith J Edwards. 2002. The Spiritual Assessment Inventory. Journal of the Scientific Study of Religion 41: 341-57.

Hill, Peter R., and Leslie J. Francis. 2005. The relationships of religiosity and personality with suicidal ideation. Mortality 10: 286-93.

Hoge, D.R. 1972. A validated Intrinsic Religious Motivation Scale. Journal for the Scientific Study of Religion 11: 369-76.

Hovey, Joseph D., Gabriela Hurtado, Lori R.A Morales, and Laura D. Seligman. 2014. Religion-based emotional social support mediates the relationship between intrinsic religiosity and mental health. Archives of Suicide Research 18: 376-91.

Jacobs, Jerry. 1967. A phenomenological study of suicide notes. Social Problems 15: 60-72.

Ji, Chang-Ho C., Tonya Perry, and Dora Clarke-Pine. 2011. Considering personal religiosity in adolescent delinquency. Journal of Psychology E Christianity 30: 3-15.

Koenig, Harold G., Faten Al Zaben, and Doaa Ahmed Khalifa. 2012. Religion, spirituality and mental health in the West and the Middle East. Asian Journal of Psychiatry 5: 180-82.

Krysinska, Karolina, and David Lester. 2016. Religiosity and suicidal ideation and behavior. In Religiosity: Psychological Perspectives, Individual Differences and Health Benefits. Edited by R. Coope. Hauppauge: Nova, pp. 71-85.

Krysinska, Karolina, David Lester, Jennifer Lyke, and Jozef Corveleyn. 2015. Trait gratitude and suicidal ideation and behavior. Crisis 36: 291-96.

Lawrence, R. E., M.A. Oquendo, and B. Stanley. 2016. Religion and suicide risk. Archives of Suicide Research 20: $1-21$.

Lester, David. 2006. Suicide and Islam. Archives of Suicide Research 10: 77-97.

Lester, David. 2012. Spirituality and religiosity as predictors of depression and suicidal ideation. Psychological Reports 110: 247-50.

Lester, David, and Leslie J. Francis. 1993. Is religiosity related to suicidal ideation after personality and mood are taken into account? Personality \& Individual Differences 15: 591-92.

Lester, D., and Rheeda L. Walker. 2017. Religiosity is a protective factor for suicidal ideation in European American students but not in African American students. Omega 74: 295-303.

Lewis, Christopher Alan. 2002. Church attendance and happiness among Northern Irish undergraduate students: No association. Pastoral Psychology 50: 191-95.

Liu, Eric Yang, and Harold G. Koenig. 2013. Measuring intrinsic religiosity. Mental Health, Religion E Culture 16: 215-24.

Lucchetti, Giancarlo, Alessandra Lamas Granero Lucchetti, Mario F. Peres, Frederico C. Leão, Alexander Moreira-Almeida, and Harold G. Koenig. 2012. Validation of the Duke Religion Index. Journal of Religion $\mathcal{E}$ Health 51: 579-86.

Moreira-Almeida, Alexander, Lotufo Neto Francisco, and Harold G Koenig. 2006. Religiousness and mental health: A review. Revista Brasileira de Psiquiatria 28: 242-50.

Osman, Augustine, Beverly A. Kopper, Francisco X. Barrios, Joylene R. Osman, Tricia Besett, and Marsha M. Linehan. 1996. The Brief Reason for Living Inventory for Adolescents (BRFL-A). Journal of Abnormal Child Psychology 24: 433-43.

Osman, A., C. L. Bagge, P. M. Gutierrez, L. C. Konick, B. A. Kopper, and F. X. Barrios. 2001. Suicidal Behaviors Questionnaire-Revised (SBQ-R): Validation with clinical and nonclinical samples. Assessment 8: 443-54. 
Reynolds, W.M. 1987. SIQ Form HS. Odessa: Psychological Assessment Resources.

Rife, J., and David Lester. 1997. Religiosity and psychological disturbance. Psychological Reports 81: 978.

Sisask, Merike, A. Värnik, K. Kõlves, J.M. Bertolote, J. Bolhari, N.J. Botega, A. Fleischmann, L. Vijayakumar, and D. Wasserman. 2010. Is religiosity a protective factor against attempted suicide: A cross-cultural case-control study. Archives of Suicide Research 14: 44-55.

Stack, Steven, and Augustine J. Kposowa. 2011. Religion and suicide: Integrating four theories cross-nationally. In International Handbook of Suicide Prevention: Research, Policy and Practice. Edited by R. O'Connor, S. Platt and J. Gordon. London: John Wiley, pp. 235-52.

Thalbourne, Michael A., Peter S. Delin, and Darryl L. Bassett. 1994. An attempt to construct short scales measuring manic-depressive like experience and behavior. British Journal of Clinical Psychology 33: 205-7.

Walker, Rheeda L., and Shaughna Bishop. 2005. Examining a model of the relation between religiosity and suicidal ideation in a sample of African American and White college students. Suicide E Life-Threatening Behavior 35: 630-39.

Walker, Rheeda L., Temiloia K. Salami, Sierra E. Carter, and Kelci Flowers. 2014. Perceived racism and suicide ideation. Suicide \& Life-Threatening Behavior 44: 548-59.

Wang, M. C., W. Nichols, and P. Nyutu. 2009. Examining gender differences in religious awareness, life satisfaction, and suicidal behavior among black college students. In Proceedings of the 42nd American Association of Suicidology Annual Meeting. Edited by J. R. Rogers. Washington: American Association of Suicidology, pp. 209-10.

Wu, Andrew, Wang Jing Yu, and Jia Cun Xian. 2015. Religion and completed suicide: A meta-analysis. PLoS ONE 10: e0131715.

(C) 2017 by the author. Licensee MDPI, Basel, Switzerland. This article is an open access article distributed under the terms and conditions of the Creative Commons Attribution (CC BY) license (http://creativecommons.org/licenses/by/4.0/). 